\title{
Incorporation of Ti as a Pyramidal Framework Site in the Mono-Layered MCM-56 Zeolite and its Oxidation Activity
}

\author{
Aleksandra Korzeniowska, ${ }^{[a]}$ Justyna Grzybek ${ }^{[a]}$ Wieslaw J. Roth, ${ }^{[a]}$ Andrzej Kowalczyk, ${ }^{[a]}$ \\ Piotr Michorczyk, ${ }^{[b]}$ Jiři Čejka, ${ }^{[c, d]}$ Jan Přech, ${ }^{[d]}$ and Barbara Gil ${ }^{*[a]}$
}

\begin{abstract}
MWW zeolite MCM-56 with Al atoms on the surface was functionalized with $\mathrm{Ti}$ to produce pyramidal $\mathrm{TiOH}$ groups. This was carried out by removal of Al with nitric acid, calcination and treatment with titanium diisopropoxide bis(acetylacetonate) (Ti(acac) $)_{2}$ (i-propoxide) $\left.{ }_{2}, \mathrm{Ti}(\mathrm{acac})_{2} \mathrm{OiPr}_{2}\right)$. Up to $0.7 \% \mathrm{Ti}$ was introduced. Using UV-Vis spectroscopy three types of Ti moieties were identified in the uncalcined materials - tetrahedral in the framework, 5/6-coordinated $\mathrm{Ti}$ on the surface, presumably in the vacated pyramidal sites, and oxide-like clusters. The presence of $\mathrm{TiOH}$ on the surface was indicated by in situ
\end{abstract}

measurement of UV-Vis spectra during calcination. It showed the band at $290 \mathrm{~nm}$, which disappeared at $500^{\circ} \mathrm{C}$, perhaps due to condensation with silanols. Catalytic oxidation tests were carried out with three samples containing $0.13,0.36$, and $0.70 \%$ $\mathrm{Ti}$ and methyl phenyl sulfide and cis-cyclooctene as model reactants. The study confirmed potential of the $\mathrm{TiOH}$ moieties in oxidation catalysis although their final form is not certain. Future studies will include increasing $\mathrm{Ti}$ content to enhance catalytic activity and better detection of this type of the Ti site.

\section{Introduction}

The premise of this work was to take advantage of the unique pyramidal site in the zeolite framework MWW, located on the external surface, for the purpose of functionalization with $\mathrm{Ti}$ atoms and possible generation of oxidation activity. These sites are usually occupied by $\mathrm{Si}-\mathrm{OH}$ groups but in the high Al-form, MCM-56, they are assumed to show high concentration of $\mathrm{Al}^{[1]}$ The idea was to extract these $\mathrm{Al}$ atoms and replace with $\mathrm{Ti}$.

Isolated $\mathrm{Ti}$ atoms incorporated in microporous zeolite frameworks are highly efficient as selective oxidation catalysts for various organic reactants. ${ }^{[2]}$ Zeolite Ti-MFI, designated TS- $1,{ }^{[3]}$ showed exceptional catalytic activity in oxidations with hydrogen peroxide leading to many efforts to generate Ti sites in other frameworks. The key to obtaining good catalysts is the synthesis itself because the capacity for incorporation of Ti into a framework may be adversely affected by various factors and

[a] A. Korzeniowska, J. Grzybek, Prof. Dr. W. J. Roth, A. Kowalczyk, Dr. B. Gil Faculty of Chemistry

Jagiellonian University

Gronostajowa 2,Kraków 30-397 (Poland)

E-mail: gil@chemia.uj.edu.pl

[b] Dr. P. Michorczyk

Faculty of Chemical Engineering and Technology

Cracow University of Technology

Warszawska 24, Kraków 31-155 (Poland)

[c] Prof. Dr. J. Čejka

Institute of Physical Chemistry

Academy of Sciences of the Czech Republic, v.v.i.

Dolejškova 2155/3, Prague 818223 (Czech Republic)

[d] Prof. Dr. J. Čejka, Dr. J. Přech

Department of Physical and Macromolecular Chemistry

Faculty of Science

Charles University

Hlavova 8, Prague 212843 (Czech Republic)

- This manuscript is part of the Anniversary Issue in celebration of 10 years of ChemCatChem. quite often a separated titania phase has been obtained. ${ }^{[4]}$ Admittedly, very reliable procedures have been developed for preparation of various Ti-zeolites but alternative approaches have been constantly pursued. ${ }^{[2,5]}$ One example is the exploitation of 2-dimensional (2D) zeolites in various layered forms which are attractive for post-synthesis modifications such as structural expansion and topotactic chemical change. ${ }^{[6]}$ Framework MWW has been synthesized with $\mathrm{Ti}$ as both $3 \mathrm{D}$ and $2 \mathrm{D}$ forms showing promising catalytic performances. ${ }^{[7]}$ One of the $2 \mathrm{D}$ MWW forms, designated MCM-56, appears to have a unique feature, namely pyramidal $\mathrm{Al}$ atoms on the surface of monolayers as shown in Figure 1. The equivalent $\mathrm{SiOH}$ sites, which are believed to populate MWW with higher Si/Al are shown for comparison. These sites can be exploited for insertion of isolated hetero-atoms such as Ti by generation of vacancies in the framework, e.g. by acid extractions of $\mathrm{Al}$, and subsequent metal atom incorporation. ${ }^{[8]}$ Herein, we present results of the study to activate MCM-56 with Ti by this method and describe characterizations to elucidate nature of the incorporated Ti species including catalytic testing.

There are several different forms of $\mathrm{Ti}$ species that can be formed upon post-synthesis treatment of zeolites ${ }^{[9]}$ - from the most desired framework substituted atoms, through different forms of oxo- or hydroxo- species formed upon hydrolysis of $\mathrm{Ti}$ precursors and their consecutive calcination, to a separate phase of $\mathrm{TiO}_{2}$, usually identified as anatase.

In the case of the aforementioned highly valued TS-1 catalyst, synthesized first by the ENI Group, a specific form of the framework titanium, called a 'closed site', is claimed to be the active center for catalytic reactions. It is a $\mathrm{Ti}$ atom coordinated by four oxygen atoms with no hydrogen attached to any of them. If an $\mathrm{OH}$ group is formed, the site is called 'open site' and is considered to be a structural defect, lowering performance of the catalyst. ${ }^{[10]}$ For the TS-1 structure, the 
amount of closed sites is limited and the $\mathrm{Ti} /(\mathrm{Ti}+\mathrm{Si})$ ratio is usually not greater than $0.025(\mathrm{Si} / \mathrm{Ti}$ around 40$) .{ }^{[1]}$

Both of these forms of Ti sites can be formed in the MWW zeolite but, as mentioned, this framework contains a special Tsite located only at the external surface of the MWW layers. It can produce pyramidal Ti center, not as defect (cf. Figure 1). It was suggested ${ }^{[7]}$ that this form may not be stable and convert upon heating into extra-framework $\mathrm{TiO}_{2}$ clusters, which are generally not desirable for oxidation reactions because they decompose $\mathrm{H}_{2} \mathrm{O}_{2}$. We can assume, based on our previous work on the incorporation of Ce into the MWW framework, ${ }^{[12]}$ that these pyramidal sites may be preferentially occupied by $\mathrm{Ti}$, since the $\mathrm{Ti}^{4+}$ cation is much bigger than $\mathrm{Si}^{4+}$ so it is more likely that it will be located at the external surfaces of the layers than in the more constrained internal parts of the framework.

In this work Ti-MWW zeolites were obtained by the treatment of MCM-56 with concentrated nitric acid to create vacancies through dealumination, followed by treatments with soluble Ti-compounds. Uncalcined MCM-56 was used as the starting material. This way the template protected inside of the micropore structure and the vacancies could be formed preferentially on the external surfaces of the layers. It was expected that $\mathrm{Al}$ atoms populating the pyramidal centers
(XRD), porosity by nitrogen sorption, concentration of acid sites by FTIR, nature of Ti sites by UV-Vis and, finally, catalytic activity in model reactions - oxidation of methyl phenyl sulfide (MPS) and cis-cyclooctene by $\mathrm{H}_{2} \mathrm{O}_{2}$.

\section{Sample Pretreatment - Dealumination and Ti-Insertion}

The starting MCM-56 consisting primarily of individual MWW mono-layers was used in as-synthesized form, containing the hexamethyleneimine ( $\mathrm{HMI})$ template. It was first treated with concentrated nitric acid for 4 hours at room temperature. The organic template (HMI) was expected to protect the framework $\mathrm{Al}$ atoms inside micropores and inhibit their extraction. At the same time the external Al atoms, especially those in the pyramidal positions were expected to be removed preferentially. Both XRD patterns shown in Figure 2 and textural properties listed in Table 1 show very little difference before and after treatment with regard to apparent structure and pore characteristics. XRF results (Table 1) show an increase in the $\mathrm{Si} /$ Al ratio from 10 to $15 / 1$, consistent with only partial removal of Al atoms. The BAS concentrations also indicate that approximately $1 / 3$ of $A \mathrm{I}$ initially present was extracted. The introduc-

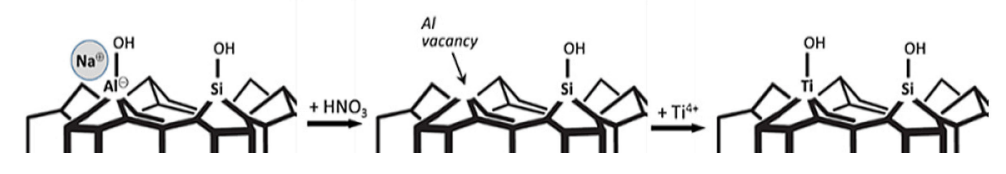

Figure 1. Formation of the Al-vacancy and pyramidal $\mathrm{Ti}$ site from pyramidal $\mathrm{Al}$ site upon acid treatment followed by reaction with $\left.\mathrm{Ti}_{(\mathrm{acac}}\right)_{2} \mathrm{OiPr}{ }_{2}$.

would be extracted and subsequently replaced with $\mathrm{Ti}$, thus predominantly forming the pyramidal Ti-atoms. The stability of incorporated Ti-sites was examined both in thermal and acidleaching treatments.

\section{Results and Discussion}

The goal of this study was substitution of $\mathrm{Ti}$ atoms into the unique pyramidal position on the surface of the framework MWW after dealumination of MCM- 56 by contacting with nitric acid. The obtained products were characterized to determine basic properties and nature of the Ti centers including catalytic oxidation. To that effect the evaluated characteristics included preservation of zeolite structure by X-ray powder diffraction

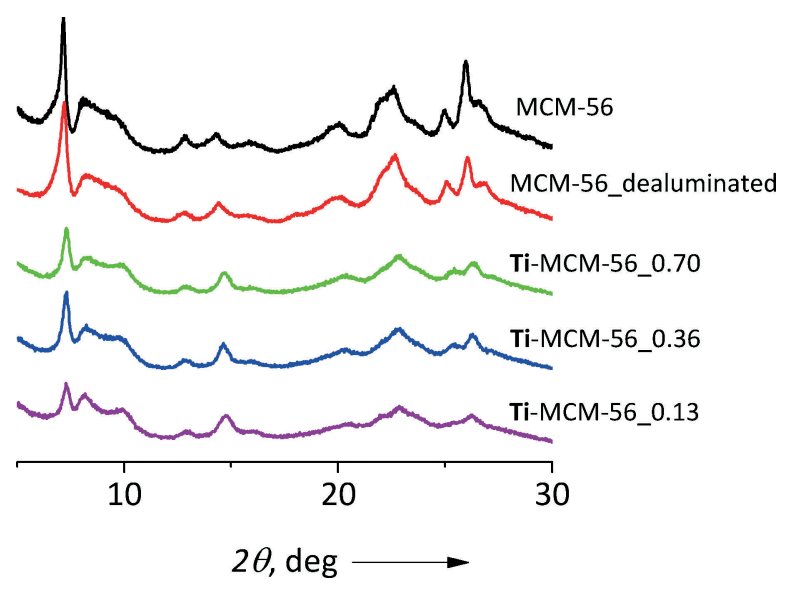

Figure 2. XRD patterns of the starting, dealuminated and Ti-treated MCM-56 zeolites in uncalcined form.

\begin{tabular}{|c|c|c|c|c|c|c|c|c|}
\hline Sample & $\mathrm{Ti}[\mathrm{wt} . \%]$ & $\mathrm{Si} / \mathrm{Al}^{[\mathrm{aj}]}$ & $\mathrm{Si} / \mathrm{Ti}^{[a]}$ & BET $\left[\mathrm{m}^{2} \mathrm{~g}^{-1}\right]$ & $\mathrm{S}_{\mathrm{ext}}\left[\mathrm{m}^{2} \mathrm{~g}^{-1}\right]$ & $\mathrm{V}_{\text {micro }}\left[\mathrm{cm}^{3} \mathrm{~g}^{-1}\right]$ & $\mathrm{BAS}^{[\mathrm{b}]}\left[\mu \mathrm{mol} \mathrm{g}^{-1}\right]$ & $\mathrm{LAS}^{[\mathrm{cc}]}\left[\mu \mathrm{mol} \mathrm{g}^{-1}\right]$ \\
\hline MCM-56 & - & 10 & - & 426 & 154 & 0.084 & 649 & 122 \\
\hline MCM-56_dealuminated & - & 15 & - & 382 & 145 & 0.063 & 428 & 74 \\
\hline TiMCM-56_0.70 & 0.70 & 15 & 100 & 365 & 148 & 0.062 & 221 & 67 \\
\hline TiMCM-56_0.36 & 0.36 & 15 & 257 & 425 & 179 & 0.081 & 371 & 97 \\
\hline TiMCM-56_0.13 & 0.13 & 16 & 525 & 360 & 171 & 0.056 & 150 & 32 \\
\hline
\end{tabular}


tion of $\mathrm{Ti}$ was carried out after calcination of the acid treated sample by contacting with $\mathrm{Ti}(\mathrm{acac})_{2} \mathrm{OiPr}_{2}$ solutions in isopropanol of different concentrations $(0.01$ and $0.001 \mathrm{M}$ for 1 hour, respectively). This gave products containing $0.70 \mathrm{wt} . \%$ of $\mathrm{Ti}$ (molar Ti:(Si+Al) equal to $1: 110)$ designated Ti-MCM-56_0.70) and $0.36 \mathrm{wt} . \%$ of $\mathrm{Ti}$ (Ti-MCM-56_0.36). Part of the $0.70 \% \mathrm{Ti}$ material was additionally treated after calcination with concentrated $\mathrm{HNO}_{3}$, which reduced the amount of $\mathrm{Ti}$ to $0.13 \%$ present only, according to UV-Vis, in tetrahedral coordination (vide infra).

XRD patterns confirmed structure preservation both of the framework and the initial layer disorder. Regarding the latter, there was a slight depression in the diagnostic band between $8-10^{\circ} 2 \theta$, indicating some lateral ordering and possibly slight condensation of the layers. ${ }^{[13]}$

The evaluation of textural properties by low-temperature nitrogen adsorption showed that dealumination did not produce change in the shape of isotherms (Figure 3), which had small hysteresis loops that may be assigned to intercrystalline, slit-like mesopores frequently observed for layered materials. There was slight decrease of BET surface area after dealumination from 426 to $380 \mathrm{~m}^{2} \mathrm{~g}^{-1}$ for treated samples and some decrease in the micropore volume, from 0.084 to $0.063 \mathrm{~cm}^{3} \mathrm{~g}^{-1}$.

The above XRD patterns and $\mathrm{N}_{2}$ adsorption isotherms indicated small changes in the structure due to acid treatment/ dealumination but FTIR revealed more significant changes in the chemical nature of the products (Figure 4). In general, for dealuminated samples, the intensity of silanol groups increased considerably, especially the hydrogen-bonded ones (IR maximum at ca. $3730 \mathrm{~cm}^{-1}$ ). The background in the whole $\mathrm{OH}$ region is elevated, suggesting greater number of defects or partial framework degradation, e.g. through breaking of $\mathrm{Si}-\mathrm{O}-\mathrm{Si}$ bonds. The band at $3615 \mathrm{~cm}^{-1}$, characteristic of $\mathrm{Si}-\mathrm{OH}-\mathrm{Al}$ groups synonymous with strong Brønsted acid sites

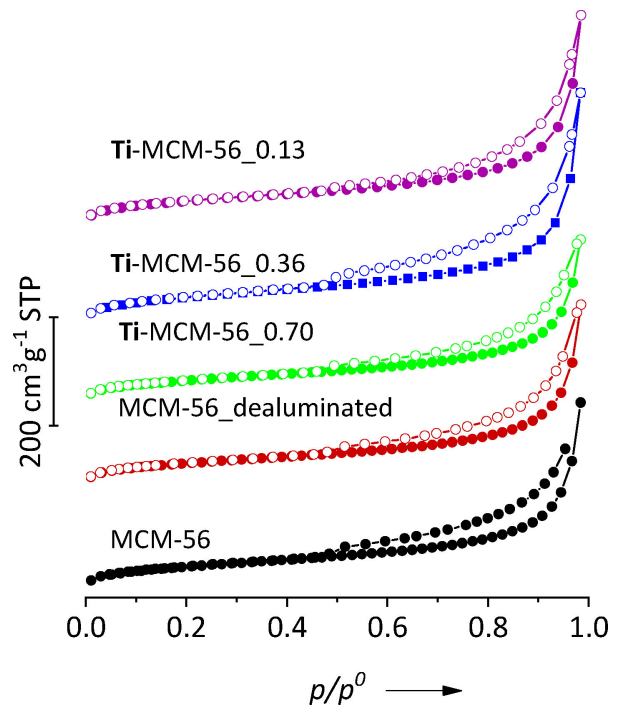

Figure 3. Nitrogen adsorption isotherms for parent, dealuminated and Tiforms of MCM- 56 zeolites. Isotherms are shifted to allow better visualization of their shape.

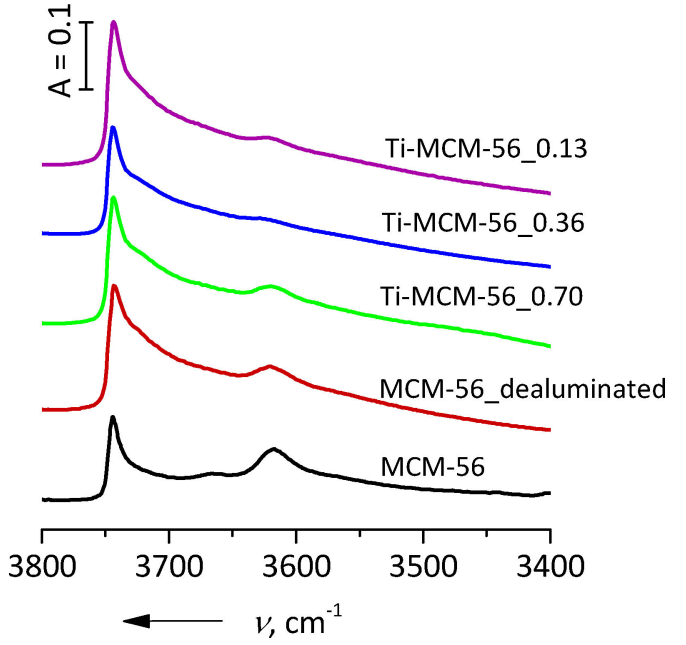

Figure 4. IR spectra of $\mathrm{OH}$ groups of the starting, dealuminated and Titreated MCM-56 zeolites.

(BAS) shows lower intensity for all treated samples. This is confirmed by the BAS concentration of ca. $450 \mu \mathrm{molg}^{-1}$, measured by pyridine desorption.

\section{Characterization and Nature of Ti}

To obtain some variation of Ti content different amounts of the Ti source were applied (cf. Scheme 1). A nominally monomeric titanium compound was used, diluted solution of $\mathrm{Ti}(\mathrm{acac})_{2} \mathrm{OiPr}_{2}$ in isopropanol, to lower the formation of multicenter $\mathrm{Ti}$ sites. No $\mathrm{TiO}_{2}$ crystallites of the sizes that can be detected with XRD were observed before and after calcination, (patterns in the range $30-70^{\circ} 2 \theta$, not shown). Concentration of BAS decreased after $\mathrm{Ti}$ incorporation by about $50 \%$ for $0.7 \%$ Ti but less than $20 \%$ for $0.36 \% \mathrm{Ti}$.

In the literature, the presence of a band at $960 \mathrm{~cm}^{-1}$ in the IR spectral region of skeletal vibrations of titanosilicates/ titanozeolites is assigned to tetrahedral $\mathrm{Ti}^{4+}$ sites $^{[11]}$ and could

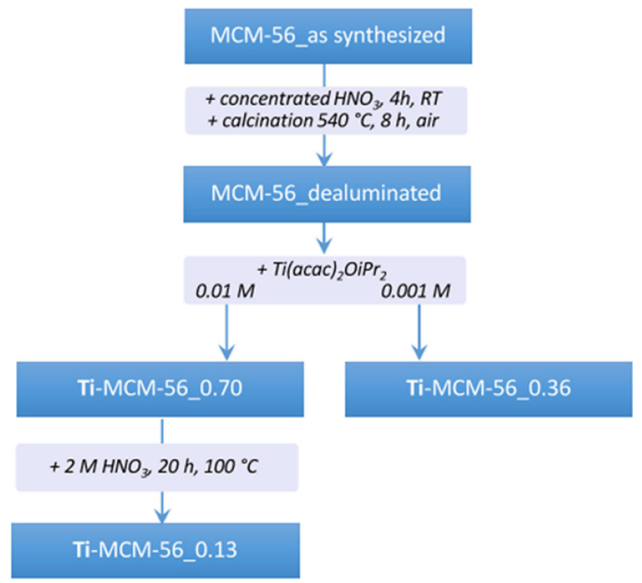

Scheme 1. Scheme of MCM-56 zeolite modifications. 
be used for its detection. However, as pointed out by Perego et al., ${ }^{[3 c]}$ Ti-free silicates or high surface area silicas have an absorption in this range as well, due to surface silanols. In our case, the dealuminated zeolites also have an absorption band in this region apparently because of having high silanol concentration. As a result, FTIR after the Ti treatment cannot verify the presence or absence of the framework fourcoordinated $\mathrm{Ti}^{4+}$ (Figure 5).

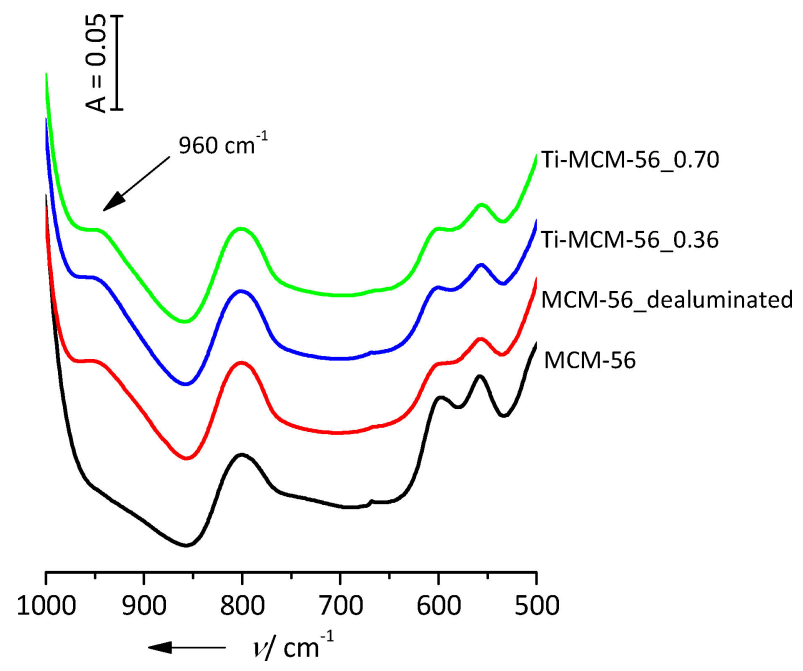

Figure 5. IR spectra of the skeletal vibrations of dealuminated and Timodified MCM- 56 zeolites. The spectra are normalized to the same intensity of the $800 \mathrm{~cm}^{-1}$ maximum (bending vibrations of $\mathrm{T}-\mathrm{O}$ bonds inside tetrahedra).

The most important aspect of the metal incorporation is the nature and quantity of different Ti-species that have been formed. The studied samples showed three different types of bands by DR-UV-Vis, with different intensities changing before and after calcination (bands at 210, 290 and $330 \mathrm{~nm}$ ). Zecchina et al. ${ }^{[4]}$ and $\mathrm{Wu}$ et al. ${ }^{[14]}$ assigned the band at $210-220 \mathrm{~nm}$ to tetrahedrally coordinated framework $\mathrm{Ti}(\mathrm{OSi})_{4}$ 'closed sites'. They are highly desirable and very active for oxidation. The absorption between 260 and $300 \mathrm{~nm}$ is characteristic of 5- and 6-coordinated extra-framework titanium species ${ }^{[15]}$ and/or partially framework $\mathrm{TiOOH}$ species (analogous to the $\mathrm{AlOOH}$ groups in boehmite). ${ }^{[16]}$ This band is also present in the MFI TS-1B material, synthesized by Balducci et al. ${ }^{[17]}$ or TS- $1+$ zeolite synthesized by $\mathrm{L}$. Wu et al. ${ }^{[18]}$ It was suggested by $\mathrm{Wu}$ et al. ${ }^{[7]}$ and Liu et al. ${ }^{[19]}$ that this band is characteristic of Ti forming octahedrally coordinated species on the external surface of the layers and identified as 'open sites'. The pyramidal Ti-OH species that might form on the MCM-56/MWW layer should give rise to the maximum at the same region, since its coordination would be very similar to any of such 'open sites'. The absorption above $300 \mathrm{~nm}$, in the case of Ti-MCM-56 zeolites at $330 \mathrm{~nm}$, is characteristic for anatase-related $\mathrm{TiO}_{2}$ phase, ${ }^{[20]}$ which is generally undesired.

The spectrum of uncalcined sample with the highest $\mathrm{Ti}$ amount, i.e. $0.70 \%$, is dominated by the maximum at $290 \mathrm{~nm}$, which indicates substantial insertion into vacated $\mathrm{Al}$ sites as
$\mathrm{TiOH}$ or its precursors. This was examined further by in situ calcination discussed later. Both samples that were reacted with $\mathrm{Ti}$ compounds contain a $\mathrm{TiO}_{2}$ phase indicated by the shoulders above $300 \mathrm{~nm}$ in UV-Vis. They are presumably present as small clusters rather than distinct anatase crystallites, since they have not been detected by XRD. The UV-Vis spectra and accordingly the nature of Ti-species changed dramatically upon calcination (as discussed above, FTIR spectra in the skeletal vibration range cannot confirm unequivocally the nature of Ti inserted in zeolite frameworks).

The changing nature of Ti in MWW due to calcination was monitored by performing calcination in situ in an UV-Vis spectrometer (Figure 7). The signal at $290 \mathrm{~nm}$ became more intense initially, reaching maximum around $250^{\circ} \mathrm{C}$ but then gradually disappeared at the highest calcination temperature $\left(500^{\circ} \mathrm{C}\right)$. This behavior is interpreted as associated with pyramidal Ti-sites, which may be at first coordinated by water molecules and alkoxy ligands in addition to their own $\mathrm{OH}$ group, thus having expanded 5- or 6-coordination sphere. Heating should cause dehydration and dehydroxylation of such species producing change in the coordination sphere and increasing tetrahedral $\mathrm{Ti}-\mathrm{OH}$ population. Subsequently the $290 \mathrm{~nm}$ peak starts to diminish and ultimately becomes rather weak. The $330 \mathrm{~nm}$ band also disappears at higher temperature and the only detectable Ti moieties by UV-Vis are tetrahedral. This indicates that the pyramidal Ti-sites do not form $\mathrm{TiO}_{2}$ during calcination, which is different from the earlier proposal. ${ }^{[7]}$ It is most probable that the species with the $290 \mathrm{~nm}$ band convert eventually into closed tetrahedral sites as they are the only ones visible in the 'working catalyst', i.e. the one activated before catalytic reaction. It is not certain how this change in $\mathrm{Ti}$ environment takes place but one possibility is condensation with $\mathrm{SiOH}$ on adjacent layers.

An additional effect at higher temperatures is the appearance of new bands in the range $395-450 \mathrm{~nm}$. They are assigned to coke being formed upon decomposition of the deposited $\mathrm{Ti}$ moieties with residual acac or i-propoxide groups. The calcination is carried out in this experiment in an inert gas flow or under vacuum and this may promote coke deposits since there is no oxygen for the combustion of organic residue. In the literature several corresponding UV-Vis signals have been reported ${ }^{[21]}$ and all of the bands visible in the spectra presented in Figure 6 with wavenumbers above $350 \mathrm{~nm}$ may be attributed to coke. Additionally, the sample with highest concentration of Ti (Ti-MCM-56_0.7) was, after vacuum activation to $500^{\circ} \mathrm{C}$, exposed to pure oxygen, and this resulted in removal of the coke (confirmed by IR) and disappearance of the 395-450 nm UV-Vis bands.

An additional piece of evidence that in the calcined samples, $\mathrm{Ti}^{4+}$ cations are incorporated in the zeolite framework is provided by the concentration of Lewis acid sites measured by pyridine adsorption. It did not change after Ti incorporation and according to Zecchina et al. ${ }^{[22]}$ Lewis acidity cannot be associated with framework $\mathrm{Ti}^{4+}$ ions. Tetrahedrally coordinated $\mathrm{Ti}^{4+}$ cations have no charge and are coordinatively saturated thus cannot interact with pyridine as Lewis acid sites. Extraframework titanium (in the form of single sites or $\mathrm{TiO}_{2}$ clusters) 


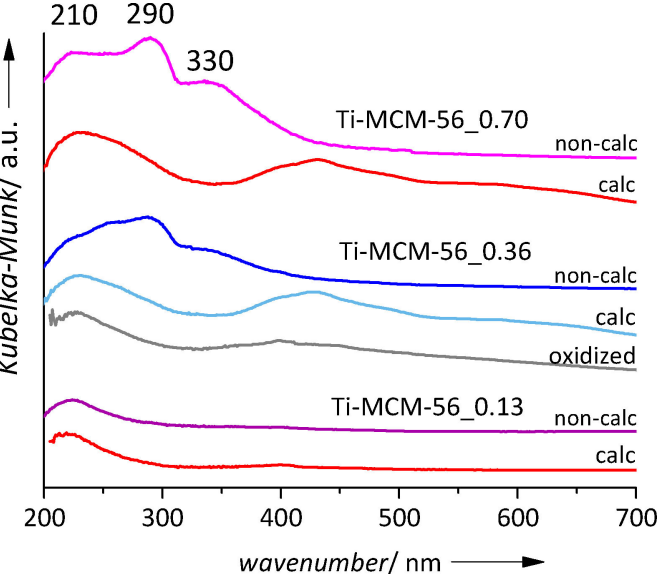

Figure 6. DR-UV-Vis spectra of Ti-MCM-56 zeolites recorded before calcination (non-calc), after calcination and exposure to air for several days (calc) and after treatment in pure $\mathrm{O}_{2}$ at $500^{\circ} \mathrm{C}$ (oxidized). The spectra are vertically offset for better visibility.
Ti-MCM-56_0.70

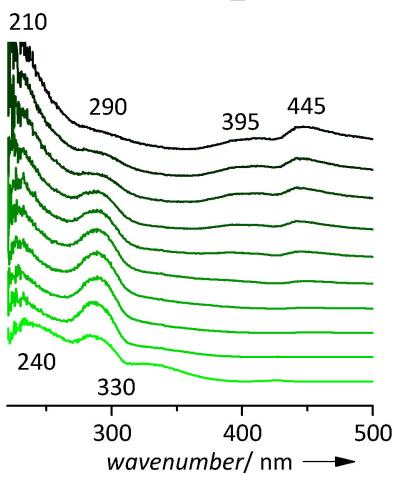

Ti-MCM-56_0.36

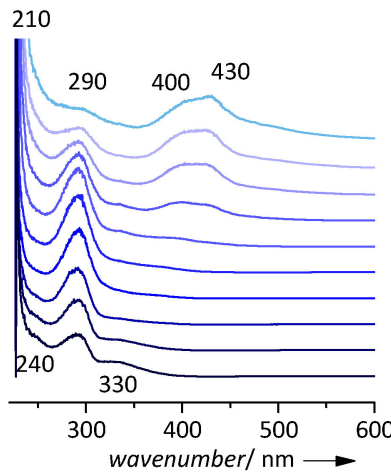

Figure 7. DR-UV-Vis spectra of Ti-MCM-56 zeolites during in situ calcination from 50 to $500^{\circ} \mathrm{C}$, step $50^{\circ} \mathrm{C}$ (from bottom to top).

may be coordinatively unsaturated thus can act as a Lewis acid site and increase their concentration. In other words, if Ti had ended outside the framework it would have increased the concentration of Lewis sites.

An additional transformation was carried out and it involved treatment of Ti-MCM-56_0.7 with acid. It resulted in disappearance of the 290 and $>330 \mathrm{~nm}$ UV-Vis absorption and only the $\mathrm{Ti}(\mathrm{OSi})_{4}$ tetrahedral sites remained (measured concentration $0.13 \%)$. As a result, the overall catalytic ability was reduced but produced more favorable selectivity in the oxidation of ciscyclooctene.

\section{Oxidation of Methyl Phenyl Sulfide and Cis-Cyclooctene Epoxidation}

The obtained Ti-MWW catalysts were tested in oxidation of methyl phenyl sulfide (MPS) and cyclooctene with aqueous hydrogen peroxide in acetonitrile at $30^{\circ} \mathrm{C}$ (MPS) and $60^{\circ} \mathrm{C}$ (cyclooctene). The conversion and yields of both reactions are summarized in Table 2. The former was chosen because it is one of the most common model compounds to study oxidation of organic sulfides. A blank experiment without the catalyst confirmed slow reaction under the experimental conditions ( $2.5 \%$ conversion after $120 \mathrm{~min}$ ). Consequently, the observed increased conversions can be attributed to the presence of catalysts. The maximum theoretical conversion is $50 \%$ under the applied conditions. ${ }^{[5 b]}$ The main product in the sulfoxidation reaction was methyl phenyl sulphoxide and only a small amount of methyl phenyl sulphone was formed (up to about $20 \%$ of the combined product amount). The activity of samples, in terms of the substrate conversion, increase with the total amount of incorporated Ti-species: $41 \%$ for the catalyst having 0.70 wt. $\%$ of Ti, $25 \%$ for 0.36 wt. $\%$ of Ti and $18 \%$ for 0.13 wt.\% of Ti. It is not possible to tell how much different Ti forms contribute to this activity but the tetrahedral sites ( $220 \mathrm{~nm}$ UV peak) seem to be particularly active - the last materials with $0.13 \%$ Ti shows only 2 times lower conversion in comparison to the most active material with 5 times higher $\mathrm{Ti}$ content. Conversion of methyl phenyl sulphoxide vs time on stream is presented in Figure 8. The product yields show similar profiles (not included for brevity) indicating that oxidation to sulfone is harder than the first step to sulfoxide.

In the case of epoxidation of cyclooctene, the conversion and $\mathrm{Ti}$ content are proportional for the two extreme concentration - 12 and $2 \%$ conversions for 0.7 and $0.13 \% \mathrm{Ti}$. The middle one with $9 \%$ conversion is closer to the most active. This may be because in this reaction, the substrate (ciscyclooctene) and the main product (cyclooctene oxide) are relatively big so it is expected that the reaction may proceed mainly at the external surfaces of the MWW layers. For that reason, even if the concentration of titanium drops by half, it is mostly retained on the surface, so the conversion dropped only from 12 to $9 \%$. For the acid-leached sample, where apparently only tetrahedral sites, located inside the micropores are present,

\begin{tabular}{|c|c|c|c|c|c|c|}
\hline \multirow[t]{2}{*}{ Catalyst } & \multirow[t]{2}{*}{ Substrate } & \multirow[t]{2}{*}{ Time $[\mathrm{min}]$} & \multirow[t]{2}{*}{ Conversion [\%] } & \multicolumn{3}{|c|}{ Yield [\%] } \\
\hline & & & & Sulphoxide & Sulphone & Cyclooctene oxide \\
\hline Ti-MCM-56_0.70 & methyl phenyl sulfide & 120 & 41 & 35 & 9 & - \\
\hline Ti-MCM-56_0.36 & methyl phenyl sulfide & 120 & 29 & 25 & 5 & - \\
\hline Ti-MCM-56_0.13 & methyl phenyl sulfide & 120 & 20 & 18 & 1 & - \\
\hline Ti-MCM-56_0.70 & cis-cyclooctene & 240 & 12 & - & - & 5 \\
\hline Ti-MCM-56_0.36 & cis-cyclooctene & 240 & 9 & - & - & 4 \\
\hline Ti-MCM-56_0.13 & cis-cyclooctene & 240 & 2 & - & - & 2 \\
\hline
\end{tabular}




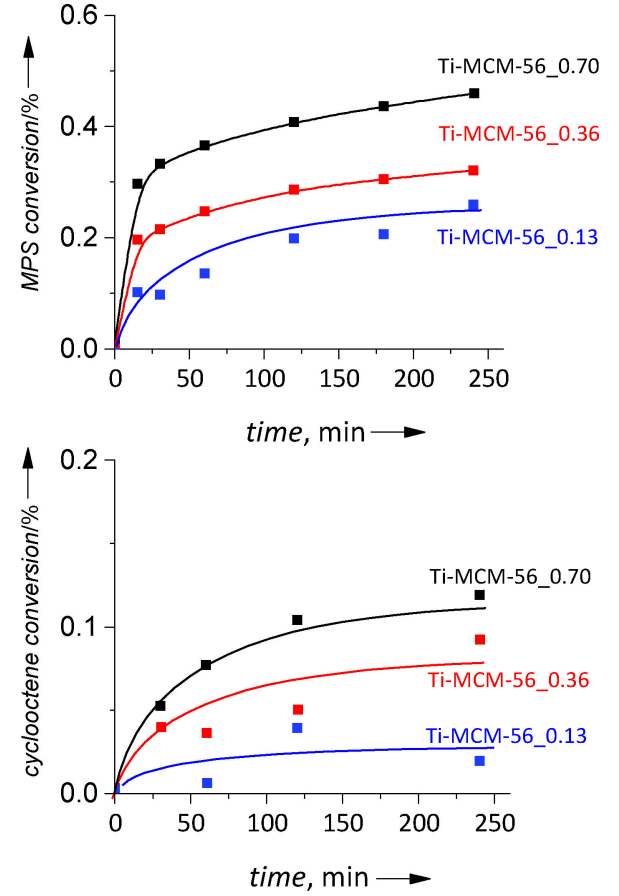

Figure 8. Conversion vs. time on stream for methyl phenyl sulfide and cyclooctene oxidation with $\mathrm{H}_{2} \mathrm{O}_{2}$ over Ti-MCM-56 zeolites.

the conversion drops to $2 \%$. At the same time Ti-MCM-56_0.13 seems to show better selectivity but it may be only due to low overall conversion with uncertain measurement error. The described catalytic tests confirm that the synthesized Ti-MCM56 materials are active in oxidation reactions, which is especially evidenced by the increasing conversion with $\mathrm{Ti}$ content. The latter effect (relative increase) seems to depend on the reactant being oxidized. This can be seen with Ti-MCM-56_0.13, which is relatively more active in MPS oxidation in comparison to the more Ti-rich catalysts but with cis-cyclooctene shows comparable conversion-to-Ti ratio to Ti-MCM-56_0.7.

The most likely mechanism in these reactions should be the same as the standard titanosilicate catalyzed oxidations, which are rather well elucidated. ${ }^{[23]}$ In this mechanism titanium atoms activate coordinated $\mathrm{H}_{2} \mathrm{O}_{2}$ by withdrawing electrons from the $\mathrm{O}-\mathrm{O}$ bond making it more susceptible to nucleophilic attack by a substrate, which leads to its oxidation. The mechanism is believed to be of the Rideal-Eley type and is not dependent on substrate adsorption on the catalyst. ${ }^{[2]}$

\section{Conclusions}

The high Al version of zeolite MWW, MCM-56, was treated with nitric acid and lost about $1 / 3$ of its Al preferentially from the surface where they were not protected by the SDA molecules. The values of Si/Al ratio changed from $10 / 1$ to $15 / 1$ and the number of Brønsted acid sites decreased by approximately the same amount, while the textural properties and structure were affected only slightly if at all. Subsequent treatment with
$\mathrm{Ti}(\mathrm{acac})_{2} \mathrm{OiPr}_{2}$ in iso-propanol was carried out leading to introduction of up to $0.7 \%$ of Ti into the MWW zeolite.

Based on UV-Vis spectra we identified 3 modes of $\mathrm{Ti}$ tetrahedral in the framework, 5/6-coordinated Ti on the surface, presumably in the vacated pyramidal sites, and oxide-like clusters. After calcination the bands due to the latter two were not seen because of conversion to tetrahedral centers on the surface terminated with $\mathrm{OH}$.

Catalytic oxidation was tested using 3 samples with different amount of Ti: $0.7,0.36$ and $0.13 \%$ and different $\mathrm{Ti}$ speciation. The conversion of methyl phenyl sulfide and ciscyclooctene increased with the amount of Ti. The sample containing $0.13 \% \mathrm{Ti}$, which was obtained by additional treatment with nitric acid after Ti-insertion showed highest relative conversion of methyl phenyl sulfide per Ti content. The study confirms potential of the open $\mathrm{TiOH}$ moieties in oxidation catalysis although their final form is not certain as it may condense to fully closed Ti centers.

\section{Experimental Section}

\section{Synthesis}

High Al-MWW zeolite (MCM-56) was prepared according to the published procedures ${ }^{[25]}$ using Ultrasil (Evonik), sodium aluminate (Riedel-de Haën ), $50 \% \mathrm{NaOH}$ and hexamethyleneimine (HMI, Sigma Aldrich) in the following molar ratio: $\mathrm{Si} / \mathrm{Al}=12, \mathrm{OH} / \mathrm{Si}=0.15$, $\mathrm{HMI} / \mathrm{Si}=0.35$ and $\mathrm{H}_{2} \mathrm{O} / \mathrm{Si}=19.3$. The gel mixture was crystallized for 37 hours in a Teflon-lined autoclave at $143^{\circ} \mathrm{C}$. The product was filtered, washed with distilled water and dried at room temperature overnight.

\section{Modification}

The $\mathrm{Ti}$ insertion procedure was adopted from the work of Baran et al. ${ }^{[26]}$ In the first step, $1 \mathrm{~g}$ samples of as-synthesized MCM-56 were stirred at $500 \mathrm{rpm}$ with $15 \mathrm{ml}$ of concentrated $\mathrm{HNO}_{3}$ acid (Avantor, Poland, p.p.a.) at room temperature for 4 hours. The solid was isolated by filtration, washed with distilled water and dried at room temperature (RT) for overnight.

The acid treated, partially dealuminated samples weighing $0.7 \mathrm{~g}$ were contacted with $50 \mathrm{ml}$ of a monomeric source of titanium: $0.01 \mathrm{M}$ and $0.001 \mathrm{M}$ solution of di-isopropoxytitanium bis (acetylacetonate) ( $\left.\mathrm{Ti}_{(\mathrm{acac}}\right)_{2} \mathrm{OiPr}_{2}$ ) in isopropanol (containing $0.24 \mathrm{~g}$ and $0.024 \mathrm{~g}$ of Ti total). These mild conditions were chosen to reduce hydrolysis and agglomeration of titanium. The obtained products were washed with isopropanol and dried at RT overnight.

Part of the Ti-MCM- 56 with $0.7 \%$ Ti content was stirred under reflux at $100^{\circ} \mathrm{C}$ with $2 \mathrm{M} \mathrm{HNO}_{3}$ for 20 hours according to the procedure by $\mathrm{Wu}$ et al. ${ }^{[27]}$ in order to remove weakly bonded metal. The solid was filtered, washed with distilled water, dried at RT overnight and calcined at $600^{\circ} \mathrm{C}$.

\section{Characterization}

X-ray powder diffraction (XRD) patterns were collected on a Philips $X^{\prime}$ Pert diffractometer APD with CuK $\alpha$ radiation $(\lambda=0.154 \mathrm{~nm})$ in the range $3-30^{\circ} 2 \theta$ with steps of $0.02^{\circ} 2 \theta$. 
Relative content of $\mathrm{Ti}, \mathrm{Al}$ and $\mathrm{Si}$ was determined in the samples formulated into pellets, $13 \mathrm{~mm}$ in diameter, with the use of EnergyDispersive XRF spectrometer (Thermo Scientific, ARL QUANT'X). The X-rays of 4-50 kV (1 kV step) with the beam size of $1 \mathrm{~mm}$ were generated with the Rh anode. The detector used was a $3.5 \mathrm{~mm}$ $\mathrm{Si}(\mathrm{Li})$ drifted crystal with a Peltier cooling (ca. $185 \mathrm{~K}$ ). For quantitative analysis, calibration with a series of metallic standards and a UniQuant software were used.

Nitrogen adsorption isotherms were determined by the standard method at $-196^{\circ} \mathrm{C}$ (liquid nitrogen temperature) using an ASAP 2025 (Micromeritics) static volumetric apparatus. Before the adsorption experiments samples were outgassed at $350^{\circ} \mathrm{C}$ using turbomolecular pump to remove pre-adsorbed water.

The DR-UV-Vis spectroscopy was carried out using an Evolution 600 (Thermo) spectrophotometer. The measurements of non-calcined samples were performed at room temperature in the range 200$900 \mathrm{~nm}$ with resolution of $2 \mathrm{~nm}$.

Calcination in situ in was carried out by DR-UV-Vis using an Ocean Optics HR2000+ instrument (integration time $20 \mathrm{~ms}, 20$ scans) equipped with an Ocean Optics DH-2000 BAL halogen-deuterium light source and a high-temperature reflection probe (FCR-7UV4002-ME-HTX, $7 \times 400 \mu \mathrm{m}$ fibers). The spectra were collected within the wavelength range $225-1100 \mathrm{~nm}$ using $\mathrm{BaSO}_{4}$ as a reflection standard. The spectra are shown in the Kubelka-Munk format $\left(F(R)=(1-R)^{2} / 2 R\right.$, where $R$ stands for reflectance). A high-temperature probe was attached at the top of the quartz microreactor. The distance between the sample bed and the high-temperature probe tip was $2-3 \mathrm{~mm}$. The sample bed thickness was $4-5 \mathrm{~mm}$.

IR spectra were recorded on Perkin Elmer Frontier spectrometer equipped with DTGS detector and a single reflection diamond ATR module, working with spectral resolution of $4 \mathrm{~cm}^{-1}$. ATR spectra were normalized to the intensity of the $800 \mathrm{~cm}^{-1}$ band, characteristic of T-O internal vibrations. Acidity was investigated by FITR spectroscopy based on adsorption of pyridine used as a probe molecule. The samples were activated in the form of selfsupporting wafers for 1 hour at $450^{\circ} \mathrm{C}$ prior to the adsorption of pyridine (POCh Gliwice, analytical grade) at $170^{\circ} \mathrm{C}$. All spectra in absorbance mode, presented in this work have been normalized to the standard $10 \mathrm{mg}$ pellet (density $3.2 \mathrm{mg} \mathrm{cm}^{-2}$ ). The concentrations of Lewis and Brønsted acid sites were evaluated using previously reported absorption coefficients ${ }^{[25]}$ : $\varepsilon(\mathrm{LAS})=$ $0.165 \mathrm{~cm}^{2} \mu \mathrm{mol}^{-1}$, and $\varepsilon(B A S)=0.044 \mathrm{~cm}^{2} \mu \mathrm{mol}^{-1}$ and calculated from intensities of the corresponding pyridine maxima after pyridine desorption at $170^{\circ} \mathrm{C}$ to ensure complete removal of weakly adsorbed species.

The oxidations of methyl phenyl sulfide (Aldrich, 99\%, Scheme 2 top) were carried out at $30^{\circ} \mathrm{C}$ in a $25 \mathrm{ml}$ magnetically stirred round
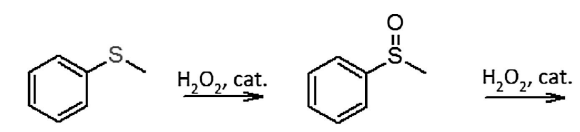

methyl phenyl sulfide methyl phenyl sulphoxide

methyl phenyl sulphone

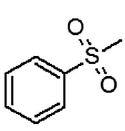

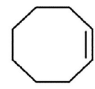

$\mathrm{H}_{2} \mathrm{O}_{2}$, cat.

cyclooctene

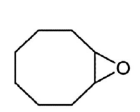

cyclooctene oxide
Scheme 2. General schemes of sulfoxidation and epoxidation reactions. bottom three-neck glass flask equipped with a Dimroth condenser (procedure taken from ref. ${ }^{[28]}$ ). Samples before reaction were activated at in air, at $450^{\circ} \mathrm{C}$ for $90 \mathrm{~min}$, cooled down in a desiccator and then transferred to the reaction flask. Typically, $8 \mathrm{mmol}(1 \mathrm{~g})$ of the sulfide was dissolved in $10 \mathrm{ml}$ of acetonitrile (Fisher chemical, HPLC grade) together with $250 \mu \mathrm{l}$ of 1,3-diisopropylbenzene as internal standard (Fluka, $95 \%$ ) and $50 \mathrm{mg}$ of a catalyst were introduced into the mixture. The substrate/catalyst mass ratio was $\mathrm{S} / \mathrm{C}=20$. The mixture was heated to the reaction temperature and the reaction was started by addition of $\mathrm{H}_{2} \mathrm{O}_{2}$ aqueous solution (Aldrich, 35 wt\%). The typically sulfide/ $\mathrm{H}_{2} \mathrm{O}_{2}$ molar ratio was equal 2 .

The conversion $(X)$ of sulfoxidation reaction was calculated as the ratio of consumed and initial molar amount of the substrate [Eq. (1)]. Yield of sulphoxide resp. sulphone was calculated as the ratio between produced sulphoxide resp. sulphone and consumed sulfide [Eq. (2)].

$\mathrm{X}=\frac{\mathrm{n}(\text { sulfide })_{\text {initial }}-\mathrm{n}(\text { sulfide })_{\text {sample }}}{\mathrm{n}(\text { sulfide })_{\text {initial }}}$

$\mathrm{y}($ product $)=\frac{\mathrm{n}(\text { product })_{\text {sample }}}{\mathrm{n}(\text { sulfide })_{\text {initial }}-\mathrm{n}(\text { sulfide })_{\text {sample }}}$

The epoxidations of cis-cyclooctene (Aldrich, 95\%, Scheme 2 bottom) were carried out according to the procedure presented by Prech et al., ${ }^{[5 a]}$ in a $25 \mathrm{ml}$ magnetically stirred glass three-necked round bottom flask equipped with a Dimroth condenser at $60^{\circ} \mathrm{C}$. The alkene/catalyst mass ratio was 10 and alkene/ $\mathrm{H}_{2} \mathrm{O}_{2}$ molar ratio was 2. Acetonitrile (Fisher chemical, HPLC grade) was used as a solvent and mesitylene ( $99 \%$, Acros-Organics) served as an internal standard. In a standard experiment, $500 \mathrm{mg}$ of cyclooctene were mixed with $250 \mathrm{mg}$ of the internal standard, $50 \mathrm{mg}$ of the catalyst and $6.65 \mathrm{ml}$ of acetonitrile. The reaction was started by addition of $\mathrm{H}_{2} \mathrm{O}_{2}$ aqueous solution (35 wt.\%, Aldrich) into the mixture. Samples were taken at regular intervals, centrifuged, cooled and analyzed using an Agilent 6850 GC system with 50 m long DB- 5 column, an autosampler and a FID or a MS detector. Helium was used as a carrier gas.

The conversion $(\mathrm{X})$ of epoxidation reaction was calculated as the ratio of consumed and initial molar amount of the substrate [Eq. (3)], while the yield was calculated by analogy to Equation (2).

$X=\frac{n\left(\text { cyclooctene }_{\text {initial }}-\mathrm{n}\left(\text { cyclooctene }_{)_{\text {sample }}}\right.\right.}{\mathrm{n}(\text { cyclooctene })_{\text {initial }}}$

\section{Acknowledgements}

Financial support from National Science Centre Poland, grant no 2016/21/B/ST5/00858 is gratefully acknowledged. J.P. and J.Č. acknowledge the Czech Science Foundation for the project P106/ 12/G015.

\section{Conflict of Interest}

The authors declare no conflict of interest. 
Keywords: Zeolites • layered compounds $\cdot$ Ti-MWW $\cdot$ pyramidal Ti-sites $\cdot$ oxidation reaction

[1] W. J. Roth, J. Cejka, R. Millini, E. Montanari, B. Gil, M. Kubu, Chem. Mater. 2015, 27, 4620-4629.

[2] a) M. G. Clerici, M. E. Domine, Liquid Phase Oxidation Via Heterogeneous Catalysis: Organic Synthesis and Industrial Applications 2013, 21-93; b) T. Tatsumi, in Modern Heterogeneous Oxidation Catalysis, Wiley-VCH Verlag $\mathrm{GmbH} \&$ Co. KGaA, 2009, pp. 125-155.

[3] a) G. Bellussi, V. Fattore, Stud. Surf. Sci. Catal. 1991, 69, 79-92; b) G. Bellussi, M. S. Rigutto, Stud. Surf. Sci. Catal. 1994, 85, 177-213; c) C. Perego, A. Carati, P. Ingallina, M. A. Mantegazza, G. Bellussi, Appl. Catal. A 2001, 221, 63-72.

[4] A. Zecchina, G. Spoto, S. Bordiga, A. Ferrero, G. Petrini, G. Leofanti, M. Padovan, Stud. Surf. Sci. Catal. 1991, 69, 251-258.

[5] a) J. Přech, J. Čejka, Catal. Today 2016, 277, 2-8; b) J. Přech, Catal. Rev. 2018, 60, 71-131.

[6] A. Corma, U. Diaz, M. E. Domine, V. Fornes, Angew. Chem. Int. Ed. 2000, 39, 1499-1501; Angew. Chem. 2000, 112, 1559-1561.

[7] P. Wu, H. Xu, L. Xu, Y. Liu, M. He, in MWW-Type Titanosilicate: Synthesis, Structural Modification and Catalytic Applications to Green Oxidations (Eds.: P. Wu, H. Xu, L. Xu, Y. Liu, M. H, Springer Berlin Heidelberg, Berlin, Heidelberg, 2013, pp. 9-34.

[8] a) J. Prech, D. Vitvarova, L. Lupinkova, M. Kubu, J. Cejka, Microporous Mesoporous Mater. 2015, 212, 28-34; b) J. P. Nogier, Y. Millot, P. P. Man C. Methivier, M. Che, S. Dzwigaj, Catal. Lett. 2009, 130, 588-592.

[9] D. Gleeson, G. Sankar, C. R. A. Catlow, J.M. Thomas, G. Spano, S. Bordiga, A. Zecchina, C. Lamberti, Phys. Chem. Chem. Phys. 2000, 2, 4812-4817.

[10] C. Lamberti, S. Bordiga, D. Arduino, A. Zecchina, F. Geobaldo, G. Spano, F. Genoni, G. Petrini, A. Carati, F. Villain, G. Vlaic, J. Phys. Chem. B 1998, 102, 6382-6390.

[11] R. Millini, E. P. Massara, G. Perego, G. Bellussi, J. Catal. 1992, 137, 497503.

[12] W. J. Roth, W. Makowski, B. Marszalek, P. Michorczyk, W. Skuza, B. Gil, J. Mater. Chem. A 2014, 2, 15722-15725.

[13] a) W. J. Roth, Stud. Surf. Sci. Catal. 2005, $158 A$ and B, 19-26; b) W. J. Roth, D. L. Dorset, Microporous Mesoporous Mater. 2011, 142, 32-36; C) K. Varoon, X. Y. Zhang, B. Elyassi, D. D. Brewer, M. Gettel, S. Kumar, J. A. Lee, S. Maheshwari, A. Mittal, C. Y. Sung, M. Cococcioni, L. F. Francis,
A. V. McCormick, K. A. Mkhoyan, M. Tsapatsis, Science 2011, 333, 72-75; d) G. G. Juttu, R. F. Lobo, Microporous Mesoporous Mater. 2000, 40, 9-23.

[14] P. Wu, T. Tatsumi, T. Komatsu, T. Yashima, J. Phys. Chem. B 2001, 105, 2897-2905.

[15] D. Srinivas, P. Manikandan, S. C. Laha, R. Kumar, P. Ratnasamy, J. Catal. 2003, 217, 160-171.

[16] M. Sasaki, Y. Sato, Y. Tsuboi, S. Inagaki, Y. Kubota, ACS Catal. 2014, 4 2653-2657.

[17] L. Balducci, D. Bianchi, R. Bortolo, R. D'Aloisio, M. Ricci, R. Tassinari, R. Ungarelli, Angew. Chem. Int. Ed. 2003, 42, 4937-4940; Angew. Chem. 2003, 115, 5087-5090.

[18] L. Z. Wu, X. J. Deng, S. F. Zhao, H. M. Yin, Z. X. Zhuo, X. Q. Fang, Y. M. Liu, M. Y. He, Chem. Commun. 2016, 52, 8679-8682.

[19] N. Liu, Y. M. Liu, W. Xie, L. L. Wang, M. Y. He, P. Wu, Stud. Surf. Sci. Catal. 2007, 170, 464-469.

[20] X. S. Liu, K. K. Lu, J. K. Thomas, J. Chem. Soc. Faraday Trans. 1993, 89, 1861-1865.

[21] M. Rutkowska, D. Macina, Z. Piwowarska, M. Gajewska, U. Diaz, L. Chmielarz, Catal. Sci. Technol. 2016, 6, 4849-4862.

[22] A. Zecchina, G. Spoto, S. Bordiga, M. Padovan, G. Leofanti, G. Petrini, Stud. Surf. Sci. Catal. 1991, 65, 671-680.

[23] a) G. H. Gao, S. F. Cheng, Y. An, X. J. Si, X. L. Fu, Y. M. Liu, H. J. Zhang, P. Wu, M. Y. He, ChemCatChem 2010, 2, 459-466; b) H. Munakata, Y. Oumi A. Miyamoto, J. Phys. Chem. B 2001, 105, 3493-3501; c) G. Ricchiardi, A. de Man, J. Sauer, Phys. Chem. Chem. Phys. 2000, 2, 2195-2204; d) S. N. Wei, H. J. He, Y. Cheng, C. P. Yang, G. M. Zeng, L. Qiu, RSC Adv. 2016, 6, 103253-103269.

[24] S. Kwon, N. M. Schweitzer, S. Park, P. C. Stair, R. Q. Snurr, J. Catal. 2015 326, 107-115.

[25] B. Gil, W. Makowski, B. Marszalek, W. J. Roth, M. Kubu, J. Čejka, Z. Olejniczak, Dalton Trans. 2014, 43, 10501-10511.

[26] R. Baran, Y. Millot, T. Onfroy, J. M. Krafft, S. Dzwigaj, Microporous Mesoporous Mater. 2012, 163, 122-130.

[27] P. Wu, T. Miyaji, Y. M. Liu, M. Y. He, T. Tatsumi, Catal. Today 2005, 99, 233-240.

[28] J. Prech, R. E. Morris, J. Cejka, Catal. Sci. Technol. 2016, 6, 2775-2786.

Manuscript received: June 18, 2018

Accepted Article published: July 30, 2018

Version of record online: August 21, 2018 